\title{
Paediatric population pharmacokinetic modelling to assess hydrocortisone replacement dosing regimens in young children
}

\author{
Robin Michelet ${ }^{1, *}$, Johanna Melin 1,2,*, Zinnia P. Parra-Guillen ${ }^{1,3}$, Uta Neumann4, Martin J Whitaker ${ }^{5}$, \\ Viktoria Stachanow ${ }^{1,2}$, Wilhelm Huisinga ${ }^{6}$, John Porter ${ }^{5}$, Oliver Blankenstein ${ }^{4}$, Richard J. Ross ${ }^{7}$ and \\ Charlotte Kloft ${ }^{1}$
}

'Department of Clinical Pharmacy and Biochemistry, Institute of Pharmacy, Freie Universitaet Berlin, Berlin, Germany, ${ }^{2}$ Graduate Research Training Program, PharMetrX, Berlin, Germany, ${ }^{3}$ Pharmacometrics and Systems Pharmacology, University of Navarra, Pamplona, Spain, ${ }^{4}$ Charité-Universitätsmedizin, Berlin, Germany, ${ }^{5}$ Diurnal Limited, Cardiff, UK, ${ }^{6}$ Institute of Mathematics, Universität Potsdam, Potsdam, Germany, and ${ }^{7}$ University of Sheffield, Sheffield, UK

*(R Michelet and J Melin contributed equally to this work)

Correspondence should be addressed to C Kloft

Email

charlotte.kloft@fu-berlin.de

\begin{abstract}
Context: Accurate hydrocortisone dosing in children with adrenal insufficiency is important to avoid the risks of over and under treatment including iatrogenic Cushing's syndrome and adrenal crisis.

Objective: To establish a population pharmacokinetic model of hydrocortisone in children and use this to refine hydrocortisone replacement regimens.

Design and methods: Pharmacokinetic study of hydrocortisone granules, available in 0.5, 1, 2 and $5 \mathrm{mg}$ dose strengths, in 24 children with adrenal insufficiency aged 2 weeks to 6 years. Cortisol concentrations quantified by LC-MS/MS were used to refine an adult pharmacokinetic model to a paediatric population model which was then used to simulate seven different hydrocortisone treatment regimens.

Results: Pre-dose cortisol levels were undetectable in $54 \%$ of the 24 children. The developed pharmacokinetic model had good predictive performance. Simulations for the seven treatment regimens using either three- or four-times daily dosing showed treatment regimens delivered an $\mathrm{AUC}_{0-24 \mathrm{~h}}$ within the $90 \%$ reference range for healthy children except in neonates where two regimens had an AUC below the 5th percentile. Cortisol concentrations at individual time points in the $24 \mathrm{~h}$ were outside the $90 \%$ reference range for healthy individuals in 50\%, 55-65\% and $70-75 \%$ for children, infants and neonates, respectively, with low cortisol levels being most prevalent.

Conclusions: Current paediatric hydrocortisone treatment regimens based on either three- or four-times daily administration replicate cortisol exposure based on $\mathrm{AUC}_{0-24 \text {, }}$, but the majority of cortisol levels are above or below physiological cortisol levels with low levels very common before the next dose.
\end{abstract}




\section{Introduction}

Patients with childhood-onset adrenal insufficiency, including the genetic disease congenital adrenal hyperplasia (CAH), have an increased morbidity and mortality in adult life that may, in part, relate to excess glucocorticoid therapy in childhood $(1,2,3,4,5,6,7$, 8). Adrenal crisis, however, is the leading cause of death in adrenal insufficiency (9), and lower hydrocortisone doses are associated with a greater risk of adrenal crisis (10). Thus, paediatricians constantly need to balance the immediate risk of adrenal crisis if they under dose children with hydrocortisone and the longerterm risk of increased morbidity and mortality due to chronic over dosing with glucocorticoids. The current recommendation for hydrocortisone replacement in children with CAH is $10-15 \mathrm{mg} / \mathrm{m}^{2} /$ day (11) split three times over the day, although the guidelines state there are insufficient data to recommend fractional distribution of doses through the day (12). An additional challenge in hydrocortisone replacement is that, until recently, there has been no appropriate formulation for paediatric dosing of hydrocortisone and children have therefore been dependent on either pharmacy or parent compounding of tablets. The most common paediatric dose prescribed is $2 \mathrm{mg}$, whereas the smallest available tablet is $5 \mathrm{mg}$ in the USA and $10 \mathrm{mg}$ in Europe (13). Hydrocortisone is poorly soluble in aqueous solutions and suspensions have been shown not to be bioequivalent to the hydrocortisone tablet (14). Furthermore, suspensions are viscous and may adsorb to equipment used in compounding (15). Studies of compounded hydrocortisone report that up to $25 \%$ of batches from pharmacies and $50 \%$ by parents were out of specification leading to clinically evident under- or over- treatment including iatrogenic Cushing's syndrome $(16,17,18)$. Hydrocortisone granules (development name Infacort) in paediatric-appropriate doses of 0.5 , 1.0, 2.0 and $5.0 \mathrm{mg}$ were licensed in Europe in 2018 (Alkindi, Diurnal Europe B.V., The Netherlands) are well tolerated, easy to administer and in a clinical study demonstrated appropriate cortisol levels in neonates, infants and children with adrenal insufficiency (19). The hydrocortisone granules have been designed for children with taste masking to cover the bitter taste of hydrocortisone and can be administered with food (20). For the first time, the availability of an accurate paediatric formulation of hydrocortisone provides us with an important tool that allows us to review hydrocortisone dosing regimens especially in young children with adrenal insufficiency.
Currently, there is limited data on hydrocortisone replacement levels in young paediatric patients ( $<6$ years of age) $(21,22)$ and no paediatric pharmacokinetic model for hydrocortisone in this age range. Other pharmacokinetic data available have been undertaken in either adults or children over the age of 5 years $(23,24,25,26,27,28)$. We have collected serum cortisol data following dosing with hydrocortisone granules in neonates, infants and children up to 6 years of age and built a paediatric population pharmacokinetic model to assess hydrocortisone replacement treatment regimens in children. Based on a previous model developed in adults (29), our aim was to develop a paediatric population pharmacokinetic model and use the predicted cortisol concentration-time profiles to analyse common treatment regimens by comparing to previously published cortisol levels in healthy children $(30,31)$.

\section{Methods}

\section{Patients and study design}

Cortisol concentrations with the aim of pharmacokinetic analysis were collected in an open label, phase 3 , single centre clinical trial conducted at the Institute of Experimental Paediatric Endocrinology at Charité-Universitätsmedizin Berlin, CVK, Berlin (EudraCT number: 2014-002265-30, ClinicalTrials.gov Identifier: NCT02720952) (19). Written informed consent was given by parents/guardian and the study was approved by the relevant independent ethics committee (Ethics committee of Berlin, No. 14/0517- EK 12). Paediatric patients with adrenal insufficiency (23 with congenital adrenal hyperplasia and 1 with hypopituitarism) in three different age groups were included: cohort 1 , 2-6 years (young children, $n=12$ patients); cohort 2 , 28 days -2 years (infants, $n=6$ ); and cohort 3 , birth-28 days (neonates, $n=6$ ). As $\mathrm{CAH}$ is the most common congenital cause of adrenal insufficiency, the majority of children recruited had CAH and there was only one with hypopituitarism, who was panhypopituitary with absolute cortisol deficiency due to congenital hypoplastic pituitary and ectopic posterior pituitary seen on MRI. Demographic details are given in Table 1 . One dose of individualised hydrocortisone granules (Alkindi ${ }^{\circledR}$, Diurnal Europe B.V., The Netherlands), corresponding to the individual standard morning dose (1-4 mg), was administered in the morning upon arrival to the clinic after at least $2 \mathrm{~h}$ of fasting. Patients were not allowed to eat within $60 \mathrm{~min}$ post-dose (30 min for children below 1 year). All patients underwent 
Table 1 Simulation scenario for children, infants and neonates after three (TID) or four (QID) times daily dosing.

\begin{tabular}{|c|c|c|c|}
\hline & Children & Infants & Neonates \\
\hline Median BW (kg) & 16.2 & 11.1 & 3.65 \\
\hline Median BSA $\left(\mathrm{m}^{2}\right)$ & 0.635 & 0.485 & 0.22 \\
\hline $\mathrm{CBG}(\mu \mathrm{g} / \mathrm{mL})$ & 28.9 & 27.0 & 27.6 \\
\hline $\begin{array}{l}\text { Initial } \\
\text { concentration }\end{array}$ & 26.2 & 9.85 & 0 \\
\hline $\begin{array}{l}\text { Recommended } \\
\text { daily dose (mg) }\end{array}$ & $\begin{array}{l}6.35-9.53 \\
(6.5-9.5)\end{array}$ & $\begin{array}{c}4.85-7.28 \\
(5-7)\end{array}$ & $\begin{array}{l}2.2-3.3 \\
(2-3.5)\end{array}$ \\
\hline TID*, (mg) & $2.5,2.5,2.5$ & $2,2,2$ & $1,1,1$ \\
\hline Daily dose (mg) & 7.5 & 6 & 3 \\
\hline \multicolumn{4}{|l|}{ TID (mg) } \\
\hline $50 \%$ & 4 & 3 & 1 \\
\hline $25 \%$ & 2 & 1.5 & 0.5 \\
\hline $25 \%$ & 2 & 1.5 & 0.5 \\
\hline Daily dose & 8 & 6 & 2 \\
\hline \multicolumn{4}{|l|}{ TID (mg) } \\
\hline $40 \%$ & 3 & 2.5 & 1 \\
\hline $20 \%$ & 1.5 & 1 & 0.5 \\
\hline $20 \%$ & 3 & 2.5 & 1 \\
\hline Daily dose & 7.5 & 6 & 2.5 \\
\hline QID*, (mg) & $2,2,2,2$ & $\begin{array}{l}1.5,1.5 \\
1.5,1.5\end{array}$ & $\begin{array}{l}0.5,0.5 \\
0.5,0.5\end{array}$ \\
\hline Daily dose & 8 & 6 & 2 \\
\hline
\end{tabular}

BW (body weight), BSA (body surface area), CBG (Corticosteroid-binding globulin); * same dose.

plasma sampling prior to dose, 1- and 4-h post-dose. Three additional samples were retrieved per patient in cohort 1 , in which every individual was randomised into one of four groups $(n=3)$ in which two extra samples were taken after 30, 45, 90, 120, 150 and/or $180 \mathrm{~min}$ and for all an extra sample around time to $C_{\min }\left(t_{\min }\right)$ was collected. Total cortisol concentrations were quantified at all these times. In addition, cortisol binding globulin (CBG) concentrations were quantified in the pre-dose sample in all patients.

\section{Measurements}

Total cortisol concentrations $\left(C_{\text {tot }}\right)$ were quantified by liquid chromatography with tandem mass spectrometry detection (LC-MS/MS) following a previously described method (13), which was adapted for the low sample volume. The linear range for $C_{\text {tot }}$ was $14.11-718.3 \mathrm{nM}$ (5.11-260.15 ng/mL) with an average imprecision of $4.75 \%$ and an average accuracy of $99.7 \%$, based on 37 quality control samples on 5 different levels. The CBG samples were diluted and quantified using ELISA (Biovendor, Brno, Czech Republic) with an LLOQ of $3.13 \mathrm{ng} / \mathrm{mL}$, intra- and inter-assay variability $<3.0 \% \mathrm{CV}$ and $<8.0 \%$ $\mathrm{CV}$, respectively (32). The bioanalysis was performed at Simbec Research Ltd (Merthyr Tydfil, UK).

\section{Population pharmacokinetic modelling}

In order to optimally analyse collected concentrations, a nonlinear mixed effects modelling approach was applied. Being the golden standard in quantitative clinical pharmacology, it allows to analyse the central tendency and variability in a dataset simultaneously and is thus ideal to apply to sparse paediatric data (33) . A previously published semi-mechanistic PK model for hydrocortisone granules with taste-masking (29) developed in healthy adults was used for the simulations in NONMEM (7.4, ICON, Dublin, Ireland) (34) and Perl speaks Nonmem (3.4.2, Uppsala University, Uppsala, Sweden) (35). This model, in which healthy adults with endogenous cortisol production received dexamethasone to suppress the hypothalamic-pituitary-adrenal (HPA) axis, consisted of a two-compartment disposition model with saturable absorption (Michaelis-Menten type) and a plasma protein binding model considering both the nonlinear binding to CBG and linear binding to albumin or erythrocytes. In addition, in adults, a constant underlying baseline was used to account for the approximately constant cortisol baseline in dexamethasone-suppressed adults. Initially, the predictive performance of the adult semi-mechanistic model was evaluated by comparing model-predicted paediatric cortisol concentration-time profiles $(n=1000)$ using the paediatric covariates (dose, body weight (BW) and $\mathrm{CBG}$ concentration) with the observed paediatric cortisol concentrations (Visual Predictive Check, VPC). Then, the adult model was investigated for optimisation to the paediatric patient population. For further analysis of the data, the log-transform-both-sides (LTBS) approach was used, meaning that the logarithms of the plasma concentrations were modelled. The first-order conditional estimation with interaction $(\mathrm{FOCE}+\mathrm{I})$ algorithm was used for parameter estimation. The development of the population model proceeded iteratively, starting from the adult model. Interindividual variability (IIV) was modelled exponentially, assuming the structural model parameters to follow a log-normal distribution, whereas the residual unexplained variability was modelled following a proportional residual error model (=additive error model in the log domain).

\section{Evaluating cortisol exposure after multiple doses of hydrocortisone granules}

A simulation-based analysis using the refined paediatric model was undertaken to visualise and evaluate differences 
in $C_{\max }, C_{\min }$ and AUC in a typical child, infant or neonate following seven treatment regimens:

- Three times daily (TID)

- Three times daily every 8 h: 07:00 h, 15:00 h and 23:00 h, with different proportions of the daily dose at the time points:

- $33 \%-33 \%-33 \%$

- $50 \%-25 \%-25 \%$

- $40 \%-20 \%-40 \%$

- Three times daily variable time: 08:00 h, 14:00 $h$, and 20:00 h, with different proportions of the daily dose at the time points:

- $33 \%-33 \%-33 \%$

- $50 \%-25 \%-25 \%$

- $40 \%-20 \%-40 \%$

- Four times daily (QID) every 6 h: 06:00 h, 12:00 h, 18:00 $h$, and 24:00 h, with equal doses at all time points

To define the reference child, the median BW, median body surface area (BSA), median CBG, and median pre-dose cortisol concentration for respective cohort (neonates, infants, and young children) were extracted from the clinical trial (see Table 1 ). The recommended hydrocortisone dose for children is $10-15 \mathrm{mg} / \mathrm{m}^{2} /$ day (11) and these targets were rounded to the nearest available dosing strengths of hydrocortisone granules. Thus, as is depicted in Table 1, the rounded doses (calculated dose for BSA in parentheses) used in the simulation for young children, infants and neonates were $6.5-9.5 \mathrm{mg}$ (6.35-9.53 mg), 5-7 mg (4.85-7.28 mg) and 2-3.5 mg (2.2-3.3 mg), respectively. The cortisol concentrationtime profiles were simulated for a typical neonate, infant and young child. The simulations were compared with previously published data from healthy children (30), from which the $90 \%$ reference range of cortisol at different clock times in healthy children were digitally extracted using WebPlotDigitizer (36). The $C_{\min }$ after $24 \mathrm{~h}$ and $\mathrm{AUC}_{0-24 \mathrm{~h}}$ were extracted from the simulated data, and the latter was also compared with the $90 \%$ reference range of prepubertal AUC from Knutsson et al. (30). Furthermore, morning (08:00-10:00 h) cortisol concentration ranges from neonates $(n=133)$, infants $(n=26)$ and children $(n=413)$ were taken from JonetzMentzel and Wiedemann (31) to provide an age-relevant comparison.

\section{Results}

\section{Population characteristics}

Twenty-four paediatric patients with adrenal insufficiency were included in the study (Table 2). The median BW and height were highest in cohort 1 (young children) and lowest in cohort 3 (neonates), whereas distribution of CBG and albumin concentrations were overlapping between the three different cohorts, indicating no agedependency for the CBG concentrations.

\section{Graphical evaluation of pharmacokinetic data}

A total of 106 cortisol samples were measured, and 54\% of the samples taken pre-dose were below the lower limit of quantification (LLOQ), including all the neonatal predose concentrations. Furthermore six samples (5.6\%) $4 \mathrm{~h}$ post-dose were below the LLOQ (14.1 nmol/L (5.11 ng/ $\mathrm{mL})$ ). The pre-dose concentrations ranged from LLOQ to $104 \mathrm{nmol} / \mathrm{L}(38 \mathrm{ng} / \mathrm{mL})$ and from LLOQ to $47.6 \mathrm{nmol} / \mathrm{L}$ $(17.4 \mathrm{ng} / \mathrm{mL})$ in infants and young children, respectively. Three very high levels at $C_{\text {max }}(>800 \mathrm{nmol} / \mathrm{L}(290 \mathrm{ng} / \mathrm{mL}))$ were observed in cohort 3 (neonates) (Fig. 1).

\section{Prediction of paediatric cortisol concentrations from adult PK model}

To evaluate the predictive performance of the semimechanistic adult PK model, the cortisol concentrations were simulated (1000 simulations) based on the covariates

Table 2 Covariates (median (range)) for paediatric patients with adrenal insufficiency (total population, $n=24$ ).

\begin{tabular}{l} 
Patient characteristics \\
\hline Female \\
Male \\
Body weight $(\mathrm{kg})$ \\
Height $(\mathrm{cm})$ \\
Age (months) \\
CBG concentrations $(\mu g / \mathrm{mL})$ \\
Morning PO dose $(\mathrm{mg})$
\end{tabular}

\begin{tabular}{c}
\hline Young children $(n=12)$ \\
\hline 5 \\
7 \\
$16.2(12.2-21.0)$ \\
$93.5(84.0-108)$ \\
$39.9(24.8-56.9)$ \\
$29.0(23.8-38.7)$ \\
$2.5(2.0-4.0)$
\end{tabular}

\begin{tabular}{c} 
Infants $(n=6)$ \\
\hline 2 \\
4 \\
$11.1(6.70-12.5)$ \\
$79.5(62.0-83.0)$ \\
$16.6(4.13-22.1)$ \\
$27.0(22.9-29.6)$ \\
$2.0(2.0-2.5)$
\end{tabular}

\begin{tabular}{c}
\hline Neonates $(n=6)$ \\
\hline 4 \\
2 \\
$3.65(2.80-4.90)$ \\
$53.5(49.0-57.0)$ \\
$0.767(0.533-0.867)$ \\
$27.6(16.8-29.6)$ \\
$2.0(1.0-2.0)$
\end{tabular}

CBG, corticosteroid-binding globulin; po, oral. 

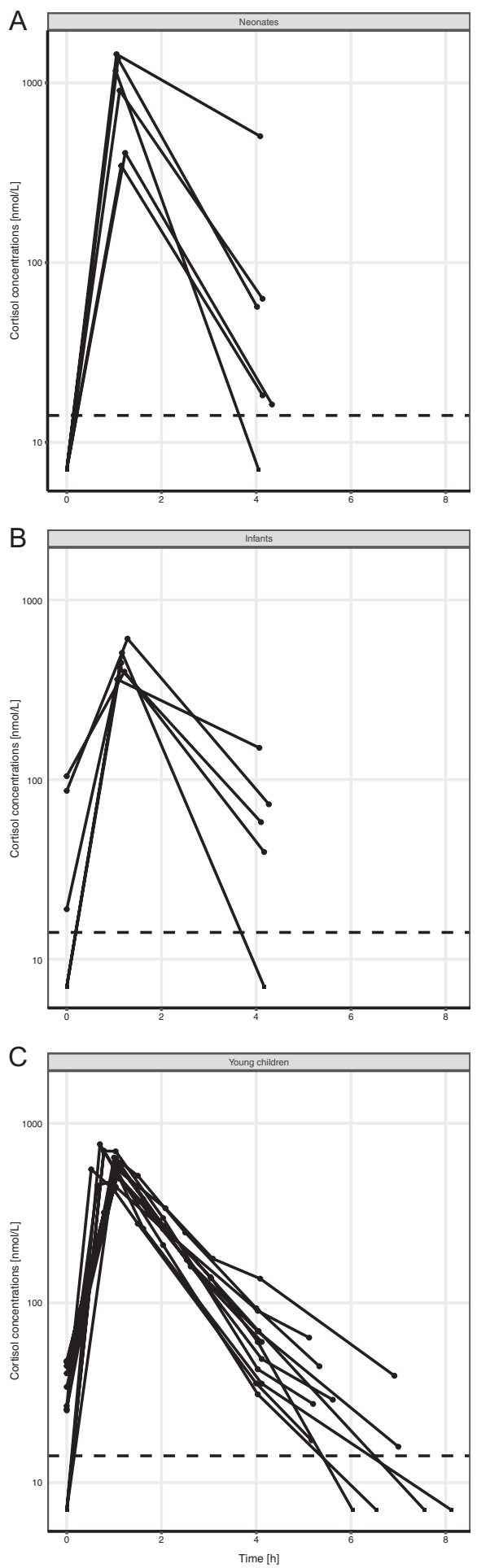

\section{Figure 1}

Absolute cortisol concentration--time profiles after single oral administration of Infacort to neonates (A), infants (B) and children (C) with adrenal insufficiency. Concentrations are depicted on a logarithmic scale and concentrations BLOQ (14.1 $\mathrm{nmol} / \mathrm{L}$, dashed line) are depicted as LLOQ/2 using squares. (dose, BW, CBG) from the paediatric patients from the current study and compared to the observed paediatric cortisol concentrations using a visual predictive check $(37,38)$. For each simulation, the median, 5th and 95th percentile were calculated per time point in order to obtain a $90 \%$ prediction interval (PI). By repeating this a 1000 times, the $95 \mathrm{CI}$ were calculated around the three simulated percentiles. Discrepancies were observed between predictions based on the adult model and the observed paediatric data (Fig. 2A): The 90\% PI lies below the median of the observations for the pre-dose concentrations and the median observed concentrations after $4 \mathrm{~h}$ lie outside the $95 \% \mathrm{CI}$ for the median simulated values indicating the need for refinement.
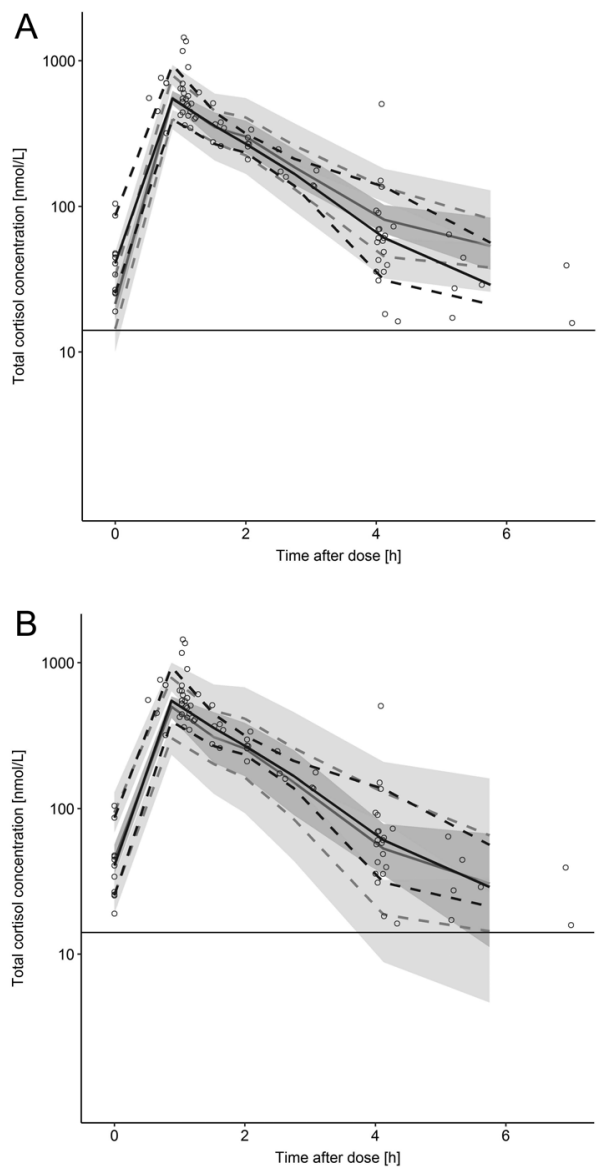

Figure 2

External model evaluation of the original developed semimechanistic adult PK model (A) and visual predictive check for the final paediatric model (B). Lines: the 5th, 50th and 95th percentiles of observed paediatric (black) and simulated paediatric (grey) data; areas: $95 \% \mathrm{Cl}$ around the simulated percentiles; circles: observations. Concentrations are depicted on a logarithmic scale. 


\section{Paediatric population pharmacokinetic model development}

As some discrepancies were observed in the prediction of paediatric cortisol concentrations using the adult PK model, it was attempted to develop a paediatric model describing the data, starting from the prior knowledge amalgamated in this adult model. For this, several adaptations on the adult model were tested. First, a constant underlying cortisol baseline (Baseline cort $_{\text {: }} 15.5$ $\mathrm{nmol} / \mathrm{L}$ ) was previously estimated based on data from dexamethasone-suppressed healthy adult volunteers. The suboptimal description of the pre-dose concentrations for the paediatric data indicated that this way of capturing the baseline needed to be re-evaluated. Using the measured pre-dose concentration (39) to inform the model improved model performance and was used during further model development. For values below the LLOQ, a baseline was estimated with the same variability as the observed pre-dose concentrations above LLOQ, assuming a shared variability for observed (above LOQ) and BLOQ baseline values. This translated to the estimation of a BLOQ population baseline parameter for the structural model and a shared stochastic interindividual variability parameter assuming lognormal distribution of the baseline value across the population.

However, re-estimating all parameters in the semimechanistic PK model based on only the sparse paediatric data resulted in an unstable model sensitive to initial estimates. Therefore, the paediatric data was pooled with the original adult data and relevant parameters were re-estimated. The original adult data was used in model development and optimisation, but not in the latter model evaluation as the scope of the current work was describing paediatric PK. Secondly, body weight which was previously added to the adult PK model using theory-based allometric scaling with fixed exponents to account for differences in body size was kept in the model (40). Thirdly, variability in the bioavailability and thus apparent clearance and central volume of distribution was included. Lastly, a maturation function to describe the age-dependent differences in clearance improved model performance slightly but was not supported enough by the sparse data, resulting in unreliable parameter estimates. The final model predicted the observed cortisol concentrations in paediatric patients over the entire concentration range as is seen in the visual predictive check, where the median, 5th and 95th percentile of the observations lie within the 95\% CI of the respective simulated percentiles (Fig. 2b). Indeed, the central tendency of cortisol concentrations was captured well, as shown by the overlap in observed and simulated median. As expected, the variability of the data was slightly overestimated, depicted by the median 5th and 95th percentile of the simulations falling outside of the observation percentiles. This is probably due to the sparse sampling and relatively low number of patients, but does not hamper model performance as the observation percentiles still fall within the $95 \% \mathrm{CI}$ of the simulation percentiles.

A schematic depiction of the final PK model and the final parameter estimates are available in Fig. 3 and Table 3, respectively, whereas the underlying model equations can be found in the Supplementary Information, see section on supplementary materials given at the end of this article. Furthermore, bootstrap (41) and log-likelihood profiling (42)

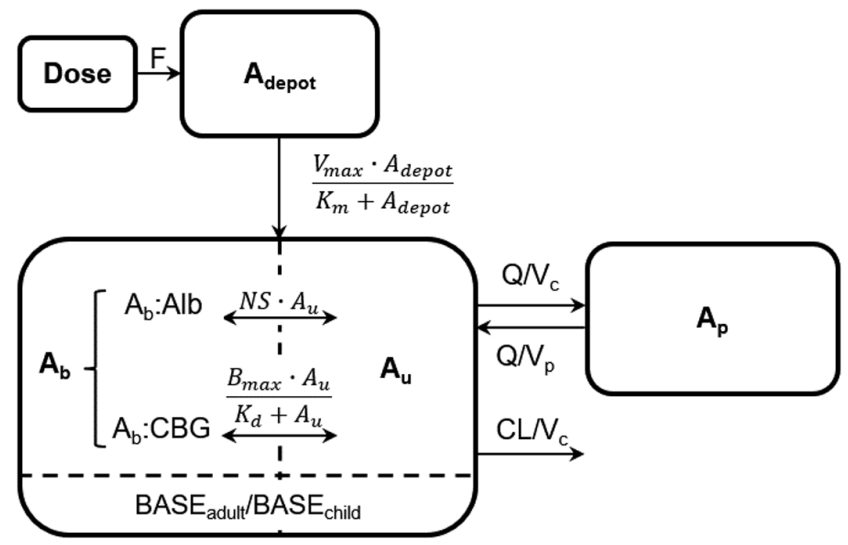

\section{Figure 3}

Schematic representation of the paediatric pharmacokinetic model. Absorption, distribution and clearance parameters were estimated using both the paediatric and adult dataset (Table 3), whereas the blood binding parameters were kept to their original value based on solely adult data (4). Scaling factor of amount in depot (F), amount in depot compartment $\left(A_{\text {depot }}\right)$, amount bound $\left(A_{\mathrm{b}}\right)$, amount bound to albumin $\left(A_{\mathrm{b}}: \mathrm{Alb}\right)$, unbound amount $\left(A_{\mathrm{u}}\right)$, amount bound to corticosteroidbinding globulin $\left(A_{\mathrm{b}}: \mathrm{CBG}\right)$, linear non-specific binding parameter (NS), maximum binding capacity $\left(B_{\max }\right)$, equilibrium dissociation constant $\left(K_{\mathrm{d}}\right)$, amount in depot compartment resulting in half of $V_{a b s, m a x}\left(K_{a b s, 50}\right)$, maximum absorption rate $\left(V_{\text {abs,max }}\right)$, apparent clearance (CL/F), apparent central volume of distribution $\left(V_{C} / F\right)$, intercompartmental clearance $(Q)$, peripheral volume of distribution $\left(V_{p}\right)$, cortisol baseline of dexamethasone suppressed healthy adults (BASE ${ }_{\text {adult }}$ ), and cortisol baseline of children with baseline measurement BLOQ $\left(\mathrm{BASE}_{\text {child,bloq }}\right)$. The dashed line divides the central compartment into the $A_{\mathrm{b}}$ and $A_{\mathrm{u}}$ subcompartments, respectively. 
Table 3 Population parameters and the respective $95 \% \mathrm{Cl}$ for the paediatric PK model.

\begin{tabular}{|c|c|}
\hline Disposition model & $\begin{array}{c}\text { Population parameter } \\
\text { estimate (RSE, CV\%) }\end{array}$ \\
\hline \multicolumn{2}{|l|}{ Fixed-effects } \\
\hline$F$ & 1 FIX \\
\hline$K_{\mathrm{abs}, 50}(\mathrm{nmol})$ & $4810(19 \%)$ \\
\hline$V_{\text {mabs, ax }}(\mathrm{nmol} / \mathrm{h})$ & $21600(10 \%)$ \\
\hline $\mathrm{CL} / F^{a}(\mathrm{~L} / \mathrm{h})$ & $409(7 \%)$ \\
\hline$V_{c} / F^{a}(\mathrm{~L})$ & $10.6(11 \%)$ \\
\hline$Q / F^{a}(\mathrm{~L} / \mathrm{h})$ & $160(18 \%)$ \\
\hline$V_{\mathrm{p}} / F^{a}(\mathrm{~L})$ & $124(14 \%)$ \\
\hline BASE $_{\text {adult }}(\mathrm{nM})$ & $15.4(6 \%)$ \\
\hline BASE $_{\text {child, bloq }}(\mathrm{nM})$ & $13.3(6 \%)$ \\
\hline \multicolumn{2}{|l|}{ Interindividual variability } \\
\hline$\omega K_{\mathrm{abs}, 50}(\mathrm{CV} \%)$ & $48.1 \%(35 \%)$ \\
\hline$\omega V_{a b s, \text { max }}(C V \%)$ & $46.0 \%(17 \%)$ \\
\hline$\omega \mathrm{CL}(\mathrm{CV} \%)$ & $19.3 \%(17 \%)$ \\
\hline$\omega \mathrm{BASE}_{\text {adult }}(\mathrm{CV} \%)$ & $34.4 \%(22 \%)$ \\
\hline$\omega F(C V \%)$ & $36.0 \%(22 \%)$ \\
\hline Residual variability & \\
\hline$\sigma \exp ^{b}(\mathrm{CV} \%)$ & $14.5 \%(8 \%)$ \\
\hline
\end{tabular}

\begin{tabular}{cc}
\hline Bootstrap median $(95 \% \mathrm{Cl})$ & LLP, 95\% Cl \\
& $\mathrm{NA}$ \\
$5030(2880-7530)$ & $3580-8550$ \\
$21900(17600-26500)$ & $1850-26000$ \\
$412(353-468)$ & $355-473$ \\
$10.6(8.58-12.6)$ & $9.31-12.0$ \\
$159(104-237)$ & $123-210$ \\
$124(84.6-163)$ & $102-150$ \\
$15.4(13.5-17.5)$ & $13.5-17.5$ \\
$13.3(12.7-13.8)$ & $11.8-15.0$ \\
$45.5 \%(12.3-117)$ & $14.1-81.8$ \\
$44.5 \%(30.2-62.2)$ & $35.1-61.8$ \\
$18.4 \%(10.6-26.2)$ & $13.5-26.5$ \\
$32.9 \%(20.0-48.0)$ & $26.3-47.2$ \\
$35.1 \%(18.3-51.6)$ & $25.9-49.3$ \\
$14.4 \%(12.5-16.9)$ & \\
\hline
\end{tabular}

Log-likelihood profiling (llp), amount in depot compartment resulting in half of $V_{\text {abs,max }}\left(K_{\text {abs,50 }}\right)$, maximum absorption rate $\left(V_{\text {abs,max }}\right)$, apparent clearance $(\mathrm{CL} / F)$, apparent central volume of distribution $\left(V_{c} / F\right)$, apparent intercompartmental clearance $(Q / F)$, apparent peripheral volume of distribution $\left(V_{p} / F\right)$,

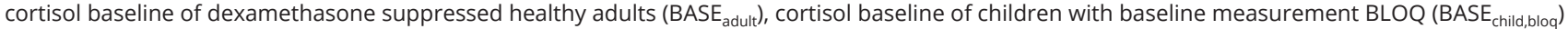

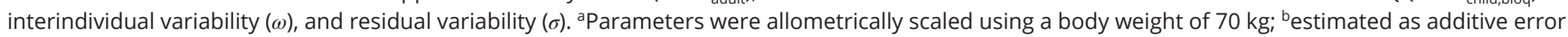
on a log scale.

CIs of the parameter estimates were constructed to confirm their robustness and precision. Just as in the adult model, a saturable absorption was incorporated, with a maximal absorption rate of $21600 \mathrm{nmol} / \mathrm{h}$ and a $K_{\mathrm{abs}, 50}$ of $4810 \mathrm{nmol}$. Most doses for both adults and children were thus below $K_{\mathrm{abs}, 50}$ and in the linear absorption range, approaching an absorption rate constant of $V_{\mathrm{abs}, \max } / K_{\mathrm{abs}, 50}=4.49 / \mathrm{h}$, which is comparable to the ratio estimated in adult data alone $(4.52 / \mathrm{h})(29) . V_{\mathrm{c}} / F$ was $10.6 \mathrm{~L}$ and $\mathrm{CL} / F$, corresponding to the unbound apparent clearance for a patient with BW of $70 \mathrm{~kg}$, was $409 \mathrm{~L} / \mathrm{h}$ with a relatively low IIV (19.3\% CV).

To assess potential age dependency in clearance, the post-hoc estimated individual apparent clearance from the refined PK model was normalised to a clearance corresponding to a $\mathrm{BW}$ of $70 \mathrm{~kg}$ and graphically compared with the adult clearance from Melin et al. (29). As seen in Fig. 4, a slightly lower clearance was estimated for neonates, which increases to adult levels for infants and children.

\section{Simulations of common hydrocortisone treatment regimens}

Using the refined paediatric population pharmacokinetic model, cortisol concentration-time simulations after commonly used three and four times daily treatment regimens were performed for a typical neonate, infant and young child as defined by the median BW, CBG concentration, and pre-dose cortisol concentration derived from patients in the current study. The $\mathrm{AUC}_{0-}$ 24h was derived and compared to previously published physiological cortisol data of Knutson et al. (30), from healthy subjects. The $\mathrm{AUC}_{0-24 \mathrm{~h}}$ was within the $90 \%$ reference range for all dosing regimens, except for two neonatal regimens, and slightly higher after QID than TID, even though the daily dose was the same (Fig. 5). Figure 6 shows the simulated cortisol concentrations in neonates, infants and young children using two of the commonly used treatment regimens with many cortisol levels falling above and below the 90\% reference range for healthy children. This is quantified in Table 4: for children, only $\sim 50 \%$ of the cortisol concentrations fell within the reference concentration interval, which dropped to $30-45 \%$ for infants and $25-30 \%$ for neonates. For children, all dosing regimens performed more or less the same in this regard, whereas for infants the irregular dosing intervals performed better than the regular ones (45\% vs 32\% inside the reference interval). For neonates the QID dosing regimen performed better than the TID (34\% vs 24-27\% inside the reference interval). The cortisol increase during the night/early morning was poorly captured for all dosing regimens. No obvious difference between selecting equal or variable doses during the day was observed. 


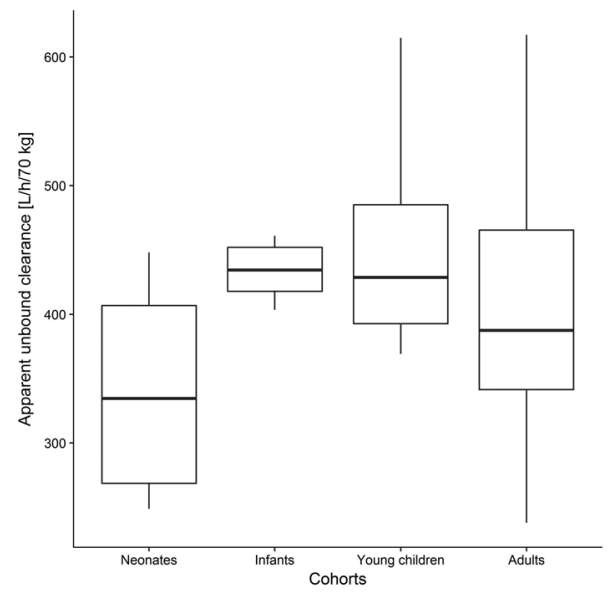

\section{Figure 4}

Derived individual apparent clearance estimates scaled to a body weight of $70 \mathrm{~kg}$ for neonates, infants, children and adults. The boxplot represents the minimum, maximum, median and interquartile range (IQR).

\section{Discussion}

We have developed a paediatric population pharmacokinetic model based on data derived from a clinical trial using a paediatric formulation of hydrocortisone that allows accurate dosing in neonates, infants and children with adrenal insufficiency. The model predicted the observed data well and was then used to examine commonly used paediatric treatment regimens. The simulations show that, although most treatment regimens replicate cumulative $24 \mathrm{~h}$ exposure to cortisol, at individual time points the majority of cortisol levels are either higher or lower than those found in healthy children and undetectable cortisol levels are common before the next predicted hydrocortisone dose. This tallies with the limited literature on hydrocortisone pharmacokinetics in children on replacement hydrocortisone that shows nonphysiological peaks and troughs are common $(43,44)$.

The initial step in the paediatric model development showed that the semi-mechanistic PK model established on adult data could relatively well predict the observed paediatric observations based on BW and CBG. The adult PK model established in dexamethasone-suppressed healthy volunteers had a constant underlying cortisol baseline to allow for an accurate description/separation of the endogenous and exogenous cortisol (29). It was apparent that this did not adequately capture the situation in paediatric patients with adrenal insufficiency, since several pre-dose concentrations were much

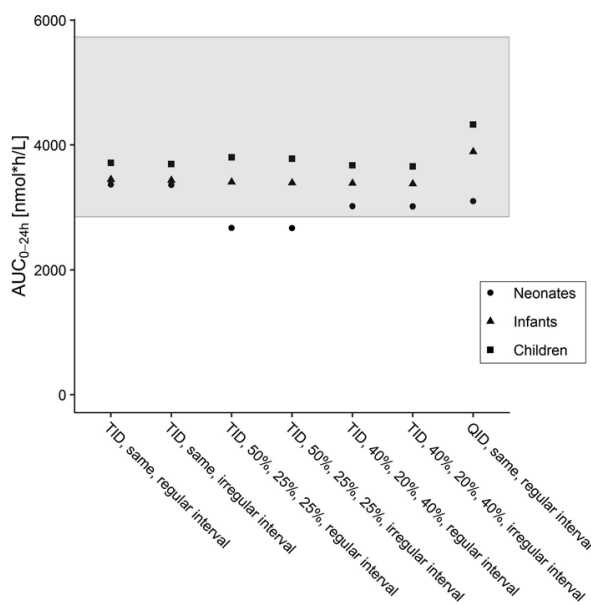

Figure 5

Simulated area under the cortisol concentration-time profile between 0 and $24 \mathrm{~h}\left(\mathrm{AUC}_{0-24 \mathrm{~h}}\right.$ ) following a four (QID) or three (TID) times daily dosing regimen in neonates, infants and children. The grey area corresponds to the $90 \%$ reference range of AUC derived from data in healthy children from Knutsson et al. (30).

higher than predicted by the cortisol baseline based on dexamethasone-suppressed adults. The observed predose cortisol concentrations could either result from the previous hydrocortisone dose, from residual cortisol synthesis, which might relate to disease severity or cyclic re-synthesis from cortisone to cortisol by 11- $\beta$ HSD (45). No pre-dose concentrations were observed for neonates, which may, in part, reflect the lack of diurnal rhythm in neonates, and in infants and children, 50\% and 75\% of the pre-dose concentrations were quantifiable. As an agedependent disease severity is unlikely, all patients were diagnosed with adrenal insufficiency and all patients were dosed every $8 \mathrm{~h}$ and the dose per $\mathrm{m}^{2}$ body surface area was the highest in neonates; these differences can only be explained by differences in pharmacokinetics in neonates compared to older children. Further studies in this vulnerable patient cohort are thus necessary. Estimating the parameters of the full semi-mechanistic PK model on paediatric data set alone resulted in a very unstable model sensitive to initial estimates, due to the relatively low number of samples per patient in the paediatric data. Including the adult data again while keeping the separate baseline and a variability on the bioavailability in mind resulted in a stable model with good parameter precision for both fixed-effect and variability parameters.

By re-estimation of the model parameters, different clearance normalised to a body weight of $70 \mathrm{~kg}$ were 


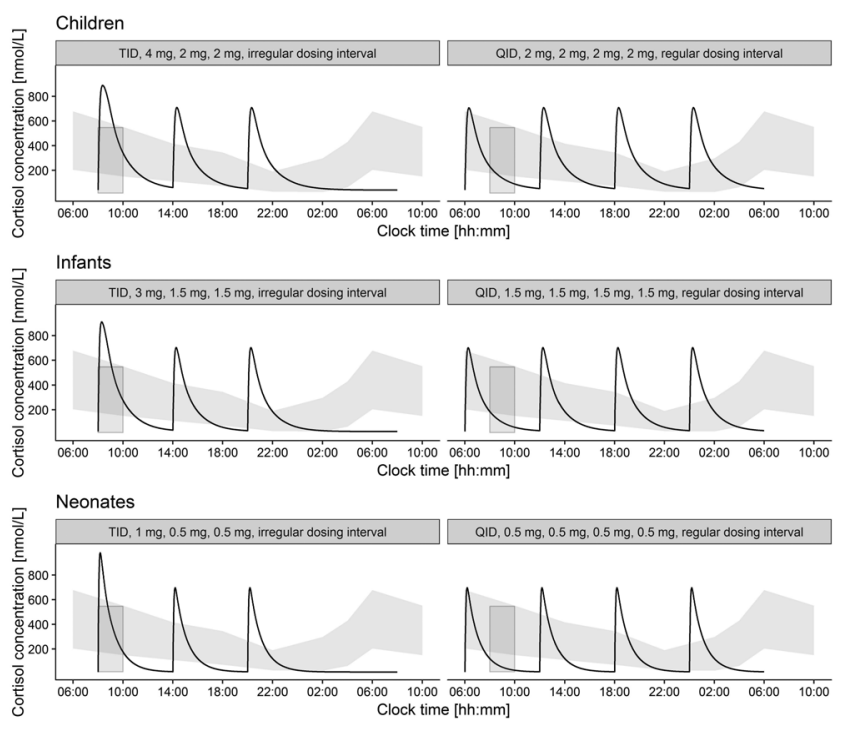

\section{Figure 6}

Simulated cortisol concentration-time profiles after three (TID) and four (QID) and times daily dosing in children, infants and neonates. The grey circadian area correspond to the $90 \%$ reference range of observed cortisol concentrations in healthy children from Knutsson et al. (30). The grey boxes refer to morning cortisol concentrations from Jonetz-Mentzel \& Wiedemann (31).

observed for the youngest population. Neonates had a lower and more variable clearance than infants, young children and adults, which can potentially be explained by the lower activity of 11- $\beta$ HSD2 (converting cortisol to cortisone) (45) and 5 $\alpha$-reductase (irreversible metabolism of cortisol to $5 \alpha$-DHF (allodihydrocortisol)) (46) in this age group. Conversely, normalised clearance in infants was more variable than in children and adults, potentially due to the high activity of $5 \alpha$-reductase in infants relative to their body size. This age-dependent pattern has been observed for many other drugs such as theophylline (47). Use of different types of maturation functions $(40,48)$ to describe this improved model performance slightly but was not supported enough by the sparse data and thus not retained in the model. Finally, body-weight adjusted clearance was similar in children (2-6 years) and adults, indicating that the PK behaviour of hydrocortisone in children of 2-6 years on is similar to that in adults suggesting that, after accounting for size, age-related differences need not to be considered when selecting dose in this population or older, as is currently the case in clinical practice.

In the current study, the optimised paediatric population pharmacokinetic model was used to simulate the typical cortisol concentration-time profiles in three representative virtual patients corresponding to the typical neonate, infant and child of the studied population. The cortisol concentration-time profiles, corresponding to proposed starting dose regimens were then visualized to see how well they corresponded to the physiological cortisol concentrations. Overall, no major differences were seen between QID and TID, but $C_{\max }$ were, in general, lower and more often within the physiological cortisol concentration range after QID compared to TID. In addition, the AUC was slightly higher after QID dosing, even though the daily dose was the same as for TID dosing. All dosing regimens failed to capture the circadian rhythm of cortisol and the majority of cortisol levels were either above or below the reference range for those reported in healthy children with low and undetectable cortisol levels being most common. Our conclusion that current dosing regimens do not reach physiological range is supported by the recent work in Melin et al. in which none of the simulated cortisol profiles could mimic the circadian rhythm of cortisol. Since that analysis was based on cortisol concentrations (measured by radioimmune

Table 4 Deviations of healthy cortisol range for children, infants and neonates after three (TID) or four (QID) times daily dosing.

\begin{tabular}{|c|c|c|c|c|c|c|}
\hline & \multicolumn{2}{|c|}{ Children, \% } & \multicolumn{2}{|c|}{ Infants, \% } & \multicolumn{2}{|c|}{ Neonates, $\%$} \\
\hline & Above & Below & Above & Below & Above & Below \\
\hline \multicolumn{7}{|l|}{ TID, regular interval } \\
\hline Same dose & 16.75 & 30.75 & 14.96 & 53.13 & 14.29 & 61.63 \\
\hline $50 \%, 25 \%, 25 \%$ & 17.13 & 28.54 & 14.63 & 53.25 & 11.25 & 64.71 \\
\hline $40 \%, 20 \%, 20 \%$ & 16.38 & 33.96 & 14.33 & 55.13 & 12.75 & 63.67 \\
\hline \multicolumn{7}{|l|}{ TID, irregular interval } \\
\hline Same dose & 14.92 & 32.50 & 12.79 & 43.29 & 12.33 & 60.33 \\
\hline $50 \%, 25 \%, 25 \%$ & 14.79 & 31.30 & 12.04 & 42.92 & 8.75 & 66.38 \\
\hline $40 \%, 20 \%, 20 \%$ & 15.04 & 34.25 & 12.50 & 45.08 & 10.71 & 62.25 \\
\hline QID, same dose, regular interval & 18.88 & 30.25 & 15.67 & 37.92 & 11.88 & 54.54 \\
\hline
\end{tabular}

Deviations are expressed as daily percentages of time above the 95th percentile or below the 5th percentile of observed cortisol concentrations in healthy children from Knutsson et al. (30). 
assay) in older children (7-17 years) administered hydrocortisone tablets, these analyses are complimentary and both support that immediate release hydrocortisone is not sufficient to replicate the circadian cortisol rhythm for children from new-born to 17 years of age.

The importance of replicating the cortisol circadian rhythm in children is not established, although there is accumulating evidence in adults that loss of the cortisol circadian rhythm, especially in congenital adrenal hyperplasia, leads to poor health outcomes (49). Prolonged hypocortisolaemia in children on hydrocortisone replacement has previously been reported $(43,44)$, but it was not associated with poor clinical outcomes in these small studies. However, a long-term study of adrenal crises in a large cohort of patients with CAH found that receiving lower hydrocortisone doses was associated with higher rates of stress dosing and illnesses and were a risk factor for adrenal crises (10). It is probable that adrenal crisis would be more likely at times of concurrent illness and physiological stress if cortisol levels are undetectable and is the rational for increasing the hydrocortisone dose during illness. Based on the data in this manuscript, current treatment regimens with hydrocortisone can neither prevent hypocortisolaemia nor replicate the circadian and ultradian rhythms of endogenous cortisol $(49,50)$.

The limitations of this study were the small number of patients studied and the relatively sparse sampling, especially in neonates. However, undertaking studies in neonates is challenging because of the short time frame to enter patients into the study and the ethical consideration of only taking samples required for clinical management. We addressed this challenge by using a population-based approach varying sampling times and taking increased number of samples in the children. For the purpose of model stability and reliable parameter estimation, all data were analysed simultaneously.

To conclude, a paediatric PK model was established based on data from paediatric patients with adrenal insufficiency. Body weight adjusted cortisol clearance was comparable for paediatric patients from 2 to 6 years and adults, indicating the need of only considering body size to select the dose in this age group. The model was used to visualise cortisol concentration-time profiles after proposed starting doses in the different age groups. On current hydrocortisone treatment regimens, cortisol concentrations remained above physiological range for $10-20 \%$ of the time and below physiological values $30-50 \%$ of the time, demonstrating a high unmet need for more physiological replacement.
Supplementary materials

This is linked to the online version of the paper at https://doi.org/10.1530/ EJE-20-0231.

\section{Declaration of interest}

VS has nothing to declare. JM is currently working at AstraZeneca in Gothenburg, Sweden. CK and WH report grants from an industry consortium (AbbVie Deutschland $\mathrm{GmbH}$ \& Co. KG, Boehringer Ingelheim Pharma GmbH \& Co. KG, Grünenthal GmbH, F. Hoffmann-La Roche Ltd, Merck KGaA and SANOFI), CK reports an additional grant from the Innovative Medicines Initiative-Joint Undertaking ('DDMoRe'), Diurnal Ltd and CK and RM report grants from the Federal Ministry of Education and Research within the Joint Programming Initiative on Antimicrobial Resistance Initiative (JPIAMR), all outside the submitted work, UN and OB reports a fee for presentation and UN grants from Diurnal Ltd, RR is Director of Diurnal Group plc, MJW is an employee of Diurnal Limited and Director of Diurnal Group plc, and JP is an employee of Diurnal Limited.

\section{Funding}

The work was carried out under a Cooperation Agreement between Freie Universitaet and Diurnal funded by the European Commission FP7 Grant (No. 281654 TAIN)

\section{Acknowledgment}

The authors thank the High-Performance Computing Service of ZEDAT at Freie Universitaet Berlin (https://www.zedat.fu-berlin.de/HPC/Home) for computing time.

\section{References}

1 Arlt W, Willis DS, Wild SH, Krone N, Doherty EJ, Hahner S, Han TS, Carroll PV, Conway GS, Rees DA, et al. Health status of adults with congenital adrenal hyperplasia: a cohort study of 203 patients. Journal of Clinical Endocrinology \& Metabolism 201095 5110-5121. (https://doi.org/10.1210/jc.2010-0917)

2 Finkielstain GP, Kim MS, Sinaii N, Nishitani M, Van Ryzin C, Hill SC, Reynolds JC, Hanna RM \& Merke DP. Clinical characteristics of a cohort of 244 patients with congenital adrenal hyperplasia. Journal of Clinical Endocrinology \& Metabolism 201297 4429-4438. (https://doi. org/10.1210/jc.2012-2102)

3 Han TS, Conway GS, Willis DS, Krone N, Rees DA, Stimson RH, Arlt W, Walker BR, Ross RJ \& United Kingdom Congenital Adrenal Hyperplasia Adult Study Executive (CaHASE). Relationship between final height and health outcomes in adults with congenital adrenal hyperplasia: United Kingdom congenital adrenal hyperplasia adult study executive (CaHASE). Journal of Clinical Endocrinology \& Metabolism 201499 E1547-E1555. (https://doi.org/10.1210/jc.20141486)

4 Hummel SR, Sadler S, Whitaker MJ, Ara RM, Dixon S \& Ross RJ. A model for measuring the health burden of classic congenital adrenal hyperplasia in adults. Clinical Endocrinology 201685 361-398. . (https://doi.org/10.1111/cen.13060)

5 Sarafoglou K, Addo OY, Turcotte L, Otten N, Wickremasinghe A, Pittock S, Kyllo J, Lteif AN, Himes JH \& Miller BS. Impact of hydrocortisone on adult height in congenital adrenal hyperplasiathe Minnesota cohort. Jurnalul Pediatrului 2014164 1141-1146.e1. (https://doi.org/10.1016/j.jpeds.2014.01.011)

6 Falhammar H, Frisén L, Hirschberg AL, Norrby C, Almqvist C, Nordenskjöld A \& Nordenström A. Increased cardiovascular and 
metabolic morbidity in patients with 21-hydroxylase deficiency: a Swedish population-based national cohort study. Journal of Clinical Endocrinology \& Metabolism 2015100 3520-3528. (https://doi. org/10.1210/JC.2015-2093)

7 Bonfig W, Roehl FW, Riedl S, Dörr HG, Bettendorf M, Brämswig J, Schönau E, Riepe F, Hauffa B, Holl RW, et al. Blood pressure in a large cohort of children and adolescents with classic adrenal hyperplasia (CAH) due to 21-hydroxylase deficiency. American Journal of Hypertension 201629 266-272. (https://doi.org/10.1093/ajh/hpv087)

8 Jenkins-Jones S, Parviainen L, Porter J, Withe M, Whitaker MJ, Holden SE, Morgan CL, Currie CJ \& Ross RJM. Poor compliance and increased mortality, depression and healthcare costs in patients with congenital adrenal hyperplasia. European Journal of Endocrinology 2018178 309-320. (https://doi.org/10.1530/EJE-17-0895)

9 Falhammar H, Frisén L, Norrby C, Hirschberg AL, Almqvist C, Nordenskjöld A \& Nordenström A. Increased mortality in patients with congenital adrenal hyperplasia due to 21-hydroxylase deficiency. Journal of Clinical Endocrinology \& Metabolism 201499 E2715-E2721. (https://doi.org/10.1210/jc.2014-2957)

10 El-Maouche D, Hargreaves CJ, Sinaii N, Mallappa A, Veeraraghavan P \& Merke DP. Longitudinal assessment of illnesses, stress dosing, and illness sequelae in patients With congenital adrenal hyperplasia. Journal of Clinical Endocrinology \& Metabolism 2018103 2336-2345. (https://doi.org/10.1210/jc.2018-00208)

11 Speiser PW, Azziz R, Baskin LS, Ghizzoni L, Hensle TW, Merke DP, Meyer-Bahlburg HFLL, Miller WL, Montori VM, Oberfield SE, et al. Congenital adrenal hyperplasia due to steroid 21-hydroxylase deficiency: an Endocrine Society clinical practice guideline. Journal of Clinical Endocrinology \& Metabolism 2010 [cited January 17, 2020] 95 4133-4160. (https://doi.org/10.1210/jc.2009-2631)

12 Speiser PW, Arlt W, Auchus RJ, Baskin LS, Conway GS, Merke DP, Meyer-Bahlburg HFL, Miller WL, Murad MH, Oberfield SE, et al. Congenital adrenal hyperplasia due to steroid 21-hydroxylase deficiency: an Endocrine Society* clinical practice guideline. Journal of Clinical Endocrinology \& Metabolism 2018103 4043-4088. (https:// doi.org/10.1210/jc.2018-01865)

13 Whitaker MJ, Spielmann S, Digweed D, Huatan H, Eckland D, Johnson TN, Tucker G, Krude H, Blankenstein O \& Ross RJ. Development and testing in healthy adults of oral hydrocortisone granules with taste masking for the treatment of neonates and infants with adrenal insufficiency. Journal of Clinical Endocrinology \& Metabolism 2015100 1681-1688. (https://doi.org/10.1210/jc.20144060)

14 Merke DP, Cho D, Anton Calis K, Keil MF \& Chrousos GP. Hydrocortisone suspension and hydrocortisone tablets are not bioequivalent in the treatment of children with congenital adrenal hyperplasia. Journal of Clinical Endocrinology \& Metabolism 200186 441-445. (https://doi.org/10.1210/jcem.86.1.7275)

15 Daniel E, Whitaker MJ, Keevil B, Wales J \& Ross RJ. Accuracy of hydrocortisone dose administration via nasogastric tube. Clinical Endocrinology 201990 66-73. (https://doi.org/10.1111/cen.13876)

16 Neumann U, Burau D, Spielmann S, Whitaker MJ, Ross RJ, Kloft C $\&$ Blankenstein O. Quality of compounded hydrocortisone capsules used in the treatment of children. European Journal of Endocrinology 2017177 239-242. (https://doi.org/10.1530/EJE-17-0248)

17 Webb EA, Watson C, Kerr S, Davies JH, Stirling H \& Batchelor H. Hydrocortisone tablets: human factors in manipulation and their impact on dosing accuracy. Endocrine Abstracts 201751 OC8.1. (https://doi.org/10.1530/endoabs.51.OC8.1)

18 Barillas JE, Eichner D, Van Wagoner R \& Speiser PW. Iatrogenic Cushing syndrome in a child With congenital adrenal hyperplasia: erroneous compounding of hydrocortisone. Journal of Clinical Endocrinology \& Metabolism 2018103 7-11. (https://doi.org/10.1210/ jc.2017-01595)

19 Neumann U, Whitaker MJ, Wiegand S, Krude H, Porter J, Davies M, Digweed D, Voet B, Ross RJ \& Blankenstein O. Absorption and tolerability of taste-masked hydrocortisone granules in neonates, infants and children under 6 years of age with adrenal insufficiency. Clinical Endocrinology 201888 21-29. (https://doi.org/10.1111/ cen.13447)

20 Daniel E, Digweed D, Quirke J, Voet B, Ross RJ \& Davies M. Hydrocortisone granules are bioequivalent when sprinkled Onto food or given directly on the tongue. Journal of the Endocrine Society 20193 847-856. (https://doi.org/10.1210/js.2018-00380)

21 Vezina HE, Ng CM, Vazquez DM, Barks JD \& Bhatt-Mehta V. Population pharmacokinetics of unbound hydrocortisone in critically ill neonates and infants with vasopressor-resistant hypotension. Pediatric Critical Care Medicine 201415 546-553. (https://doi.org/10.1097/PCC.0000000000000152)

22 Arnold JD, Rajan VS, Gross A, Veldhuis JD, Leslie G, Milmlow D \& Silink M. Pharmacokinetics of hydrocortisone in extremely premature infants in the first six weeks of life. Pediatric Research 199945 183A-183A. (https://doi.org/10.1203/00006450-199904020-01085)

23 Werumeus Buning J, Touw DJ, Brummelman P, Dullaart RPF, van den Berg G, van der Klauw MM, Kamp J, Wolffenbuttel BHR \& van Beek AP. Pharmacokinetics of oral hydrocortisone - results and implications from a randomized controlled trial. Metabolism: Clinical and Experimental 201771 7-16. (https://doi.org/10.1016/j. metabol.2017.02.005)

24 Charmandari E, Johnston A, Brook CG \& Hindmarsh PC. Bioavailability of oral hydrocortisone in patients with congenital adrenal hyperplasia due to 21-hydroxylase deficiency. Journal of Endocrinology 2001169 65-70. (https://doi.org/10.1677/ joe.0.1690065)

25 Peters CJ, Hill N, Dattani MT, Charmandari E, Matthews DR \& Hindmarsh PC. Deconvolution analysis of 24-h serum cortisol profiles informs the amount and distribution of hydrocortisone replacement therapy. Clinical Endocrinology 201378 347-351. (https://doi.org/10.1111/j.1365-2265.2012.04502.x)

26 Hindmarsh PC \& Charmandari E. Variation in absorption and half-life of hydrocortisone influence plasma cortisol concentrations. Clinical Endocrinology 201582 557-561. (available at: http://doi. wiley.com/10.1111/cen.12653). (https://doi.org/10.1111/cen.12653)

27 Derendorf H, Mollmann H, Barth J, Mollmann C, Tunn S \& Krieg M. Pharmacokinetics and oral bioavailability of hydrocortisone. Journal of Clinical Pharmacology 199131 473-476. (https://doi. org/10.1002/j.1552-4604.1991.tb01906.x)

28 Toothaker RD \& Welling PG. Effect of dose size on the pharmacokinetics of intravenous hydrocortisone during endogenous hydrocortisone suppression. Journal of Pharmacokinetics \& Biopharmaceutics 198210 147-156. (https://doi.org/10.1007/ BF01062332)

29 Melin J, Parra-Guillen ZP, Hartung N, Huisinga W, Ross RJ, Whitaker MJ \& Kloft C. Predicting cortisol exposure from paediatric hydrocortisone formulation using a semi-mechanistic pharmacokinetic model established in healthy adults. Clinical Pharmacokinetics 201757 1-13.

30 Knutsson U, Dahlgreen J, Marcus C, Rosberg S, Bronnegard M, Stierna P, Knutsson U, Dahlgren J, Marcus C, Rosberg S, et al. Circadian cortisol rhythms in healthy boys and girls: relationships with age, growth, body composition, and pubertal development. Journal of Clinical Endocrinology \& Metabolism 199782 536. (https:// doi.org/10.1210/jcem.82.2.3769).

31 Jonetz-Mentzel L \& Wiedemann G. Establishment of reference ranges for cortisol in neonates, infants, children and adolescents. European Journal of Clinical Chemistry and Clinical Biochemistry 1993 31 525-529. (https://doi.org/10.1515/cclm.1993.31.8.525)

32 Lewis JG, Lewis MG \& Elder PA. An enzyme-linked immunosorbent assay for corticosteroid-binding globulin using monoclonal and polyclonal antibodies: decline in CBG following synthetic ACTH. Clinica Chimica Acta: International Journal of Clinical Chemistry 2003 328 121-128. (https://doi.org/10.1016/s0009-8981(02)00417-5) 
33 Anderson BJ, Allegaert K \& Holford NHG. Population clinical pharmacology of children: general principles. European Journal of Pediatrics 2006165 741-746. (https://doi.org/10.1007/s00431-0060188-y)

34 Beal S, Sheiner LB, Boeckmann A \& Bauer RJ. NONMEM User's Guides 1989-2009. Ellicott City, MD, USA: Icon Development Solutions, 2009.

35 Lindbom L, Pihlgren P \& Jonsson EN. PsN-Toolkit - a collection of computer intensive statistical methods for non-linear mixed effect modeling using NONMEM. Computer Methods \& Programs in Biomedicine 200579 241-257. (https://doi.org/10.1016/j. cmpb.2005.04.005)

36 Rohatgi A. WebPlotDigitizer v.3, p 10. Texas, USA: Austin, 2016.

37 Holford N \& Karlsson MO. Model evaluation. Visual Predictive Checks, pp 1-17. Abstract: 1434, 2008. (available at: http://www. page-meeting.org/?abstract $=1434$ ).

38 Brendel K, Comets E, Laffont C, Laveille C \& Mentré F. Metrics for external model evaluation with an application to the population pharmacokinetics of gliclazide. Pharmaceutical Research 200623 2036-2049. (https://doi.org/10.1007/s11095-006-9067-5)

39 Dansirikul C, Silber HE \& Karlsson MO. Approaches to handling pharmacodynamic baseline responses. Journal of Pharmacokinetics \& Pharmacodynamics 200835 269-283. (https://doi.org/10.1007/ s10928-008-9088-2)

40 Anderson BJJBJ \& Holford NH. Mechanism-based concepts of size and maturity in pharmacokinetics. Annual Review of Pharmacology \& Toxicology 2008 [cited December 22, 2014] 48 303-332. (https://doi. org/10.1146/annurev.pharmtox.48.113006.094708)

41 Efron B. Nonparametric confidence intervals. In The Jackknife, the Bootstrap and Other Resampling Plans [Internet], pp. 75-90. Society for Industrial and Applied Mathematics, 1982. (https://doi. org/10.1137/1.9781611970319.ch10).

42 Sheiner LB. Analysis of pharmacokinetic data using parametric models. III. Hypothesis tests and confidence intervals. Journal of Pharmacokinetics \& Biopharmaceutics 198614 539-555. (https://doi. org/10.1007/BF01059660)
43 Maguire AM, Ambler GR, Moore B, McLean M, Falleti MG \& Cowell CT. Prolonged hypocortisolemia in hydrocortisone replacement regimens in adrenocorticotrophic hormone deficiency. Pediatrics 2007120 e164-e171. (https://doi.org/10.1542/peds.20062558)

44 DeVile CJ \& Stanhope R. Hydrocortisone replacement therapy in children and adolescents with hypopituitarism. Clinical Endocrinology 199747 37-41. (https://doi.org/10.1046/j.13652265.1997.2101025.x

45 Martinerie L, Pussard E, Meduri G, Delezoide AL, Boileau P \& Lombès M. Lack of renal 11 beta-hydroxysteroid dehydrogenase type 2 at birth, a targeted temporal window for neonatal glucocorticoid action in human and mice. PLoS One 20127 e31949. (https://doi. org/10.1371/journal.pone.0031949)

46 Thigpen AE, Silver RI, Guileyardo JM, Casey ML, McConnell JD \& Russell DW. Tissue distribution and ontogeny of steroid 5 alphareductase isozyme expression. Journal of Clinical Investigation 199392 903-910. (https://doi.org/10.1172/JCI116665)

47 Johnson TN, Rostami-Hodjegan A \& Tucker GT. Prediction of the clearance of eleven drugs and associated variability in neonates, infants and children. Clinical Pharmacokinetics 200645 931-956. (https://doi.org/10.2165/00003088-200645090-00005)

48 Germovsek E, Barker CIS, Sharland M \& Standing JF. Scaling clearance in paediatric pharmacokinetics: all models are wrong, which are useful? British Journal of Clinical Pharmacology 201783 777-790. (https://doi.org/10.1111/bcp.13160)

49 Porter J, Blair J \& Ross RJ. Is physiological glucocorticoid replacement important in children? Archives of Disease in Childhood $2017 \mathbf{1 0 2}$ 199-205. (https://doi.org/10.1136/archdischild-2015-309538)

50 Kalafatakis K, Russell GM, Harmer CJ, Munafo MR, Marchant N, Wilson A, Brooks JC, Durant C, Thakrar J, Murphy P, et al. Ultradian rhythmicity of plasma cortisol is necessary for normal emotional and cognitive responses in man. Proceedings of the National Academy of Sciences of the United States of America 2018115 E4091-E4100. (https://doi.org/10.1073/pnas.1714239115)

Received 13 March 2020

Revised version received 11 June 2020

Accepted 2 July 2020 